\title{
Role of substrate on nucleation and morphology of gold nanoparticles produced by pulsed laser deposition
}

\author{
V. Resta, ${ }^{1, *}$ C. N. Afonso, ${ }^{1}$ E. Piscopiello, ${ }^{2}$ and G. Van Tendeloo ${ }^{3}$ \\ ${ }^{1}$ Laser Processing Group, Instituto de Óptica, CSIC, Serrano 121, E-28006 Madrid, Spain \\ ${ }^{2}$ ENEA, Department of Advanced Physical Technologies and New Materials, Brindisi Research Center, \\ S.S. 7 Appia km 706, I-72100 Brindisi, Italy \\ ${ }^{3}$ EMAT, University of Antwerp, Groenenborgerlaan 171, B-2020 Antwerp, Belgium
}

(Received 1 December 2008; published 9 June 2009; corrected 11 June 2009)

\begin{abstract}
This work compares the morphology of gold nanoparticles (NPs) produced at room temperature on singlecrystalline (MgO nanocubes and plates) and amorphous (carbon/glass plates) substrates by pulsed laser deposition (PLD). The results show that similar deposition and nucleation rates $\left(>5 \times 10^{13} \mathrm{~cm}^{-2} \mathrm{~s}^{-1}\right)$ are achieved irrespective of the nature of the substrate. Instead, the shape of NPs is substrate dependent, i.e., quasispheres and faceted NPs in amorphous and single-crystalline substrates, respectively. The shape of the latter is octahedral for small NPs and truncated octahedral for large ones, with the degree of truncation being well explained using the Wulff-Kaichew theorem. Furthermore, epitaxial growth at room temperature is demonstrated for single-crystalline substrate. The large fraction of ions having energies higher than $200 \mathrm{eV}$ and the large flux of species arriving to the substrate $\left(10^{16}\right.$ at. $\left.\mathrm{cm}^{-2} \mathrm{~s}^{-1}\right)$ involved in the PLD process are, respectively, found to be responsible for the high nucleation rates and epitaxial growth at room temperature.
\end{abstract}

DOI: 10.1103/PhysRevB.79.235409 PACS number(s): 81.15.Fg, 61.46.Hk, 81.07. - b, 68.55.A-

\section{INTRODUCTION}

The control of the morphology (dimensions, shape, and number density) of nanoparticles (NPs) is essential for exploiting their properties in many fields. Examples include optical applications or biosensors based on the use of surface plasmon resonances that depend on the NPs morphology ${ }^{1}$ or nanocatalysis in which the catalytic action depends on both the NP shape and the nature of the support or substrate. ${ }^{2}$ However, controlling the shape of supported NPs by thinfilm technologies is generally difficult since it depends both on kinetic and thermodynamic parameters. ${ }^{3}$ The NP equilibrium shape becomes generally defined by the surface energy of the substrate if it is isotropic (typically glassy or amorphous), whereas the energy balance depends additionally on crystal orientation in the case of single-crystalline substrates. The latter has led to several studies on the production of metal NPs on single-crystalline oxides as a means to produce oriented or faceted NPs or even nanoarrays/wires. However, this approach usually requires thermal activation through heating the support. ${ }^{3-6}$

Pulsed laser deposition (PLD) is well known for its superior capability for producing epitaxial growth especially for oxides. $^{7}$ In addition, it has extensively been applied to produce metal NPs on amorphous substrates for which it is well documented that PLD leads to quasispherical NPs for low metal contents and to irregularly shaped NPs due to coalescence for higher values. ${ }^{8,9}$ The nucleation and growth mechanism of metals on dielectric substrates appear to be qualitatively similar to that of other deposition procedures such as evaporation, molecular-beam epitaxy (MBE), or sputtering, i.e., of Volmer-Weber type that happens when the atoms (or molecules) of the deposit are more strongly bound to each other than to the substrate. ${ }^{22}$ The use of PLD for producing NPs on single-crystalline substrates has much less been studied, and even when some degree of orientation has been reported, ${ }^{4}$ no shape changes have been highlighted with respect to what is observed on amorphous supports. In addition, shape and orientation are found to strongly depend on the kinetic energy of the species involved in the deposition process. $^{4,10,11}$ The idea that species with high kinetic energies promote epitaxial growth at low temperatures, even below room temperature (RT), has long time ago been proposed for the case of Ag. ${ }^{12}$

Gold has been for many years a case metal to study because, on the one hand, the related surface plasmon resonance can be tuned from the visible to the near IR range of the spectrum by changing the NPs' dimensions or shape. ${ }^{1}$ On the other hand, despite the generally considered inert character of bulk gold, gold NPs become active if they are smaller than $5 \mathrm{~nm}$ and are supported by an oxide catalyst. ${ }^{13}$ In this work, we have used $\mathrm{MgO}$ because it is considered as a model system for surface studies due to its simple rock-salt crystal and highly ionic structure, as well as its low mismatch with respect to $\mathrm{Au}$. In addition, it exhibits catalytic activity for a wide variety of reactions. Furthermore, $\mathrm{MgO}$ nanocubes have recently been synthesized and used as substrates allowing direct observation of the NPs in different orientations by transmission electron microscopy (TEM) with no further sample preparation. . $^{2,14,15}$

This work reports a comparison study of Au NPs produced simultaneously on single-crystalline $(\mathrm{MgO})$ and amorphous (carbon/glass) substrates by PLD. The ultimate aim is to investigate if PLD offers any advantage for promoting epitaxial growth or controlling the shape of metal NPs when compared to other techniques.

\section{EXPERIMENT}

The samples were produced by PLD in vacuum $\left(5 \times 10^{-6} \mathrm{mbar}\right)$ on a substrate held at room temperature and placed $35 \mathrm{~mm}$ away from the target. Both target and sub- 
strate holders were continuously rotated. The center of the substrate holder was shifted a few mm from the plasma expansion axis in order to produce a homogeneous deposit (within $5 \%$ ) in an area $>150 \mathrm{~mm}^{2}$. Three substrates were settled simultaneously on the substrate holder: a 1-mm-thick glass plate, a 1.5-mm-thick [110] MgO plate, and a copper grid covered by carbon and supporting $\mathrm{MgO}$ nanocubes. The latter was produced following the procedure described elsewhere. ${ }^{15}$

The Au NPs have been produced by focusing an ArF laser beam $(\lambda=193 \mathrm{~nm}$ and $\tau=20 \mathrm{~ns}$ full width half maximum) to a spot of $1.1 \pm 0.1 \mathrm{~mm}^{2}$ on a $99.99 \%$ Au target at an angle of $45^{\circ}$ with respect to the target normal. The laser repetition rate was set at $20 \mathrm{~Hz}$, and the fluence at the target site was $\sim 2.0 \mathrm{~J} \mathrm{~cm}^{-2}$. The number of pulses on the $\mathrm{Au}$ target was varied in order to change the amount of gold deposited on the substrates and thus produce Au NPs having different dimensions. The gold content of the specimens deposited on amorphous (glass) and single-crystalline $(\mathrm{MgO})$ plates was analyzed by Rutherford backscattering (RBS). A $2.0 \mathrm{MeV}$ ${ }^{4} \mathrm{He}^{+}$beam was used, and the experimental spectra were analyzed using the SIMNRA simulation code, with the error in the determination of the gold content being $2 \%$.

The morphology, size, and crystalline structure of Au NPs were characterized by conventional TEM and high-resolution electron microscopy (HRTEM), using samples on carbon coated grids supporting $\mathrm{MgO}$ nanocubes. The observations were performed using a TECNAI F30 transmission electron microscope operating at $300 \mathrm{kV}$ with point-to-point resolution of $0.205 \mathrm{~nm}$ and a JEOL 4000EX (400 kV) TEM with $0.17 \mathrm{~nm}$ point-to-point resolution. The image analysis was performed by studying areas of at least $200 \times 200 \mathrm{~nm}^{2}$.

\section{RESULTS}

Figure 1 shows the amount of gold [Au] deposited on amorphous and single-crystalline substrates as a function of the number of pulses used to ablate the Au target. The results show that $[\mathrm{Au}]$ follows a similar linear dependence on the number of laser pulses as reported earlier, ${ }^{8,9}$ irrespective of the substrate used.

Figure 2(a) shows a low magnification TEM image where several $\mathrm{MgO}$ nanocubes decorated by NPs can be seen. The

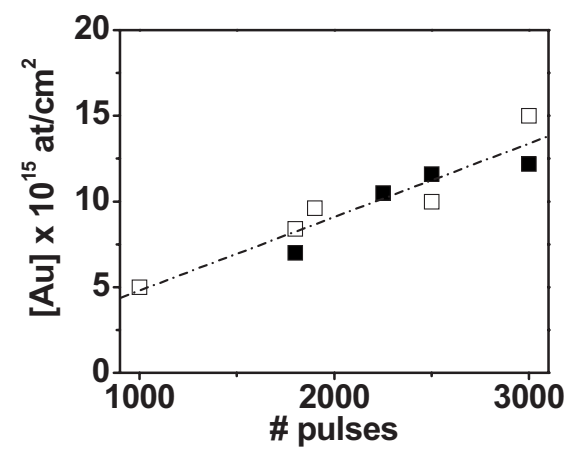

FIG. 1. Gold content of specimens on ( $\square$ ) $\mathrm{MgO}$ and ( $\square$ ) glass plates as a function of the number of laser pulses used to ablate the Au target.
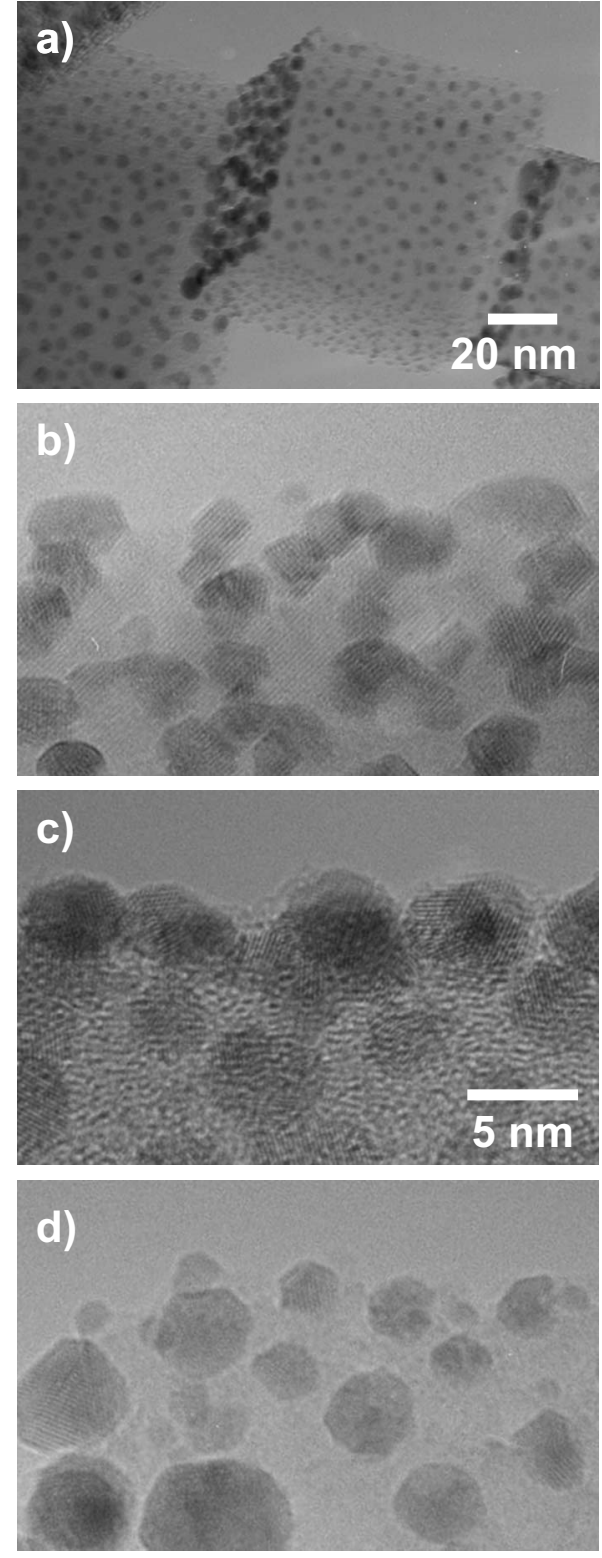

FIG. 2. (a) Low magnification TEM image of $\mathrm{MgO}$ nanocubes having Au NPs on all their faces. HRTEM images of Au NPs at the edge of (b) and (d) $\mathrm{MgO}$ nanocubes and (c) carbon for samples having increasing [Au]: (b) 7.0, (c) 9.6, and (d) 10 $\times 10^{15}$ at. $\mathrm{cm}^{-2}$.

nanocubes have an average side length of $60 \mathrm{~nm}$, and it is seen that the $\mathrm{Au}$ NPs are produced on all faces. Figures 2(b)-2(d) show images of Au NPs at increasing [Au]. Lattice fringes are always visible evidencing the crystalline character of the NPs. In all cases, the images have been taken at the edge of one $\mathrm{MgO}$ nanocube [Figs. 2(b) and 2(d)] or at a bended edge of the carbon supporting film [Fig. 2(c)]. This allows appreciation of NPs in different orientations but makes precise determination of the NPs dimension difficult due to the uncertainty on the orientation of the substrate with respect to the electron beam. In spite of these limitations, it is seen that the overall dimensions of the NPs increase as the metal content increases [from Figs. 2(b)-2(d)] as reported earlier in similar experiments. ${ }^{8,9}$ Furthermore, the compari- 

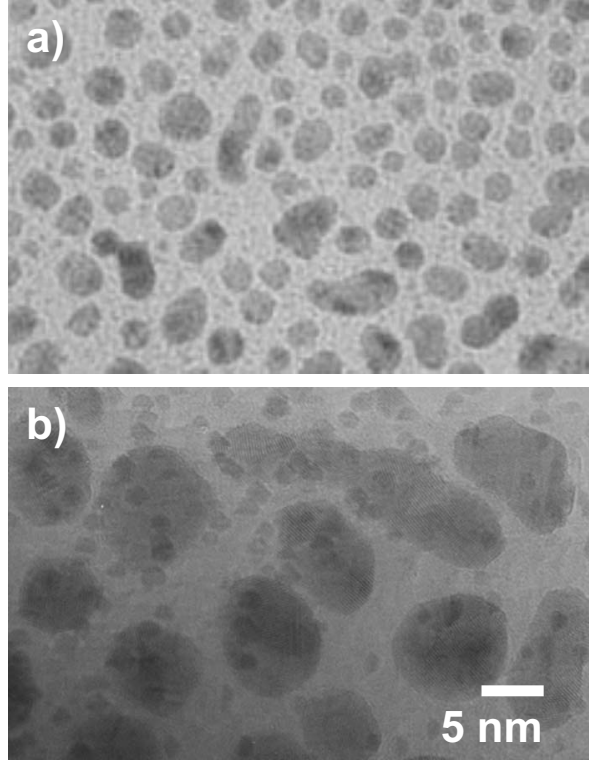

FIG. 3. HRTEM images of samples on (a) amorphous and (b) single-crystalline substrates produced simultaneously using 2240 laser pulses.

son of Figs. 2(b) and 2(d) with Fig. 2(c) makes evident that NPs on single-crystalline substrate are clearly faceted while those on amorphous substrate are quasispheres or nearly cuboctahedral as reported elsewhere for Au NPs on the surface of a Stöber silica nanosphere. ${ }^{16}$

Figure 3 shows approximately plan view images of two samples that were produced simultaneously on (a) amorphous and (b) single-crystalline substrates. It is seen that the size distribution of NPs in the former is smooth while that in the latter shows a bimodal distribution; i.e., in addition to large NPs, there are small NPs of about $2 \mathrm{~nm}$ whose image overlaps in many cases with that of the large ones. Despite the fact that the image in Fig. 3(b) has been taken with no perfect alignment of the $\mathrm{MgO}$ nanocube surface with respect to the electron beam, it is clear that the large NPs on singlecrystalline substrate are much bigger than those on the amorphous one [Fig. 3(a)]. The large NPs on the latter substrate have average in-plane diameter of $5 \pm 1 \mathrm{~nm}$.

Figure 4 shows the number density of NPs on carbon substrate as a function of $[\mathrm{Au}]$ where it is seen that it decreases as $[\mathrm{Au}]$ increases. Due to the alignment problems of

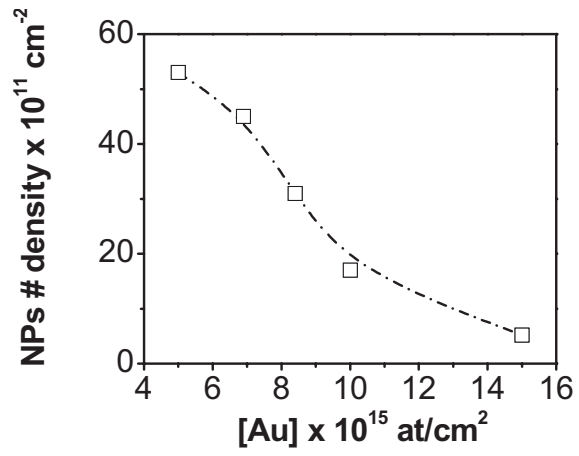

FIG. 4. Number density of Au NPs on carbon as a function of the metal content.

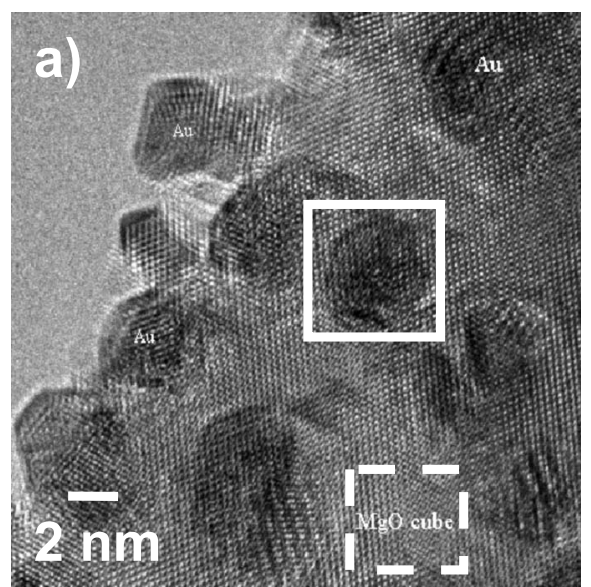

\section{b) $\mathrm{MgO}[110]$}

\section{c) $\mathrm{MgO}(002)+\mathrm{Au}(002)$}

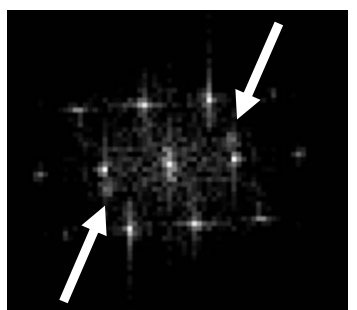

\section{d) $\mathrm{MgO}$ (002)}

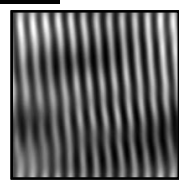

\section{Au (002)}

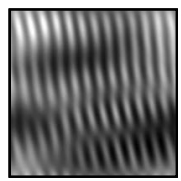

FIG. 5. (a) HRTEM image of a sample on $\mathrm{MgO}$ nanocubes having $[\mathrm{Au}]=7.0 \times 10^{15}$ at $\mathrm{cm}^{-2}$; (b) filtered image of the [110] $\mathrm{MgO}$ substrate from dashed line square in (a); (c) diffraction pattern of Au NPs on [110] MgO taken from full line square in (a); and (d) reconstructed crystal fringes for [110] $\mathrm{MgO}$ and $\mathrm{Au}$ by inverse fast Fourier transformation (FFT) of the $\mathrm{MgO}$ and $\mathrm{Au}$ (marked by arrows) spots in the pattern in (c).

the $\mathrm{MgO}$ nanocubes and the bimodal distribution of NPs observed in this case [Fig. 3(b)], the number density of NPs on $\mathrm{MgO}$ could not be precisely determined. However, the values achieved were all on the same order of magnitude $\left(20-50 \times 10^{11} \mathrm{~cm}^{-2}\right)$.

Figure 5(a) shows a HRTEM image of the sample on crystalline substrate having the lowest $[\mathrm{Au}](7.0$ $\times 10^{15}$ at $\mathrm{cm}^{-2}$ ). We have used FFT to analyze NPs orientation with respect to the substrate. The FFT of $\mathrm{MgO}$ related image in Fig. 5(b) is determined from the filtered image of an uncovered $\mathrm{MgO}$ region [area within dashed line square in Fig. 5(a)]. The diffraction pattern of the area within the full line square in Fig. 5(a) is shown in Fig. 5(c) where the (002) 


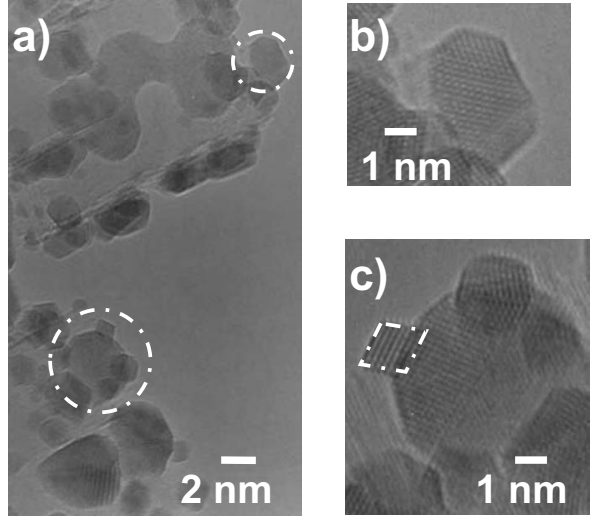

FIG. 6. (a) HRTEM image of sample on $\mathrm{MgO}$ nanocubes having $[\mathrm{Au}]=12 \times 10^{15}$ at. $\mathrm{cm}^{-2}$; (b) and (c) are details of the crosssection images of NPs highlighted by circles in (a). The area within dashed diamond in (c) highlights one of the small NPs.

$\mathrm{MgO}$ spots and the (002) Au spots (faint spots indicated by arrows) can be discriminated. Selecting the two pairs of (002) spots and calculating the inverse FFT, we obtained the images shown in Fig. 5(d) illustrating the orientation of (002) planes of both $\mathrm{MgO}$ and $\mathrm{Au}$. The comparison of these images shows that the two crystalline lattices are nearly parallel, with a $2^{\circ}$ tilt.

Figure 6(a) shows a HRTEM image of the sample on single-crystalline substrate having the highest $[\mathrm{Au}](12$ $\times 10^{15}$ at $\mathrm{cm}^{-2}$ ) and aligned in order to image cross-section views of some NPs with respect to the substrate. Figures 6(b) and 6(c) show higher magnification images of NPs within circles in Fig. 6(a) where it is further evidenced that the NPs are faceted and the small NPs are attached in many cases to the large ones. The analysis of the shape of the large NPs shows that they are truncated octahedral with different degrees of truncation. Instead, the analysis of the small ones shows that they have typically an octahedral shape [see the NP within the dashed area in Fig. 6(c)]. Furthermore, the aspect ratio of large NPs, defined as the height divided by the lateral dimension, is $<0.7$. Instead, in spite of the overlapping of images of NPs at different orientations, Fig. 2(c) evidences that the NPs on amorphous substrates are rounder and have an aspect ratio of $>0.7$, similar to earlier reports based on real cross-section images of NPs on amorphous substrates produced by a similar procedure. ${ }^{8}$

\section{DISCUSSION}

The dependence of the number density of NPs on time/ coverage, or $[\mathrm{Au}]$ as shown in Fig. 4, is typically reported to first increase for low coverage during the nucleation process and to reach a saturation value for higher coverage during pure growth process. It finally decreases once coalescence occurs, typically around $0.5 \mathrm{ML} .{ }^{5,17}$ Both the minimum $[\mathrm{Au}]$ value in this work that is significantly higher than $0.5 \mathrm{ML}$ and the decrease in the number density of NPs as a function of $[\mathrm{Au}]$ shown in Fig. 4 evidence that we are working in the coalescence regime. In spite of being in this regime, the minimum number density of NPs $\left(50 \times 10^{11} \mathrm{~cm}^{-2}\right)$ is ap- proximately 1 order of magnitude higher than the saturation value reported for gold irrespective of the substrate nature or temperature ${ }^{5,17,18}$ Interestingly, an intermediate value (16 $\times 10^{11} \mathrm{~cm}^{-2}$ ) was reported for a similar amount of $[\mathrm{Au}] \mathrm{de}-$ posited on amorphous carbon using ion-beam deposition. ${ }^{19}$ Values in the range of $3-5 \times 10^{11} \mathrm{~cm}^{-2}$ were reported for gold produced by Knudsen cell evaporation on $\mathrm{MgO}$ at $600{ }^{\circ} \mathrm{C}$ (Ref. 5) or by electron-beam evaporation on $\mathrm{KBr}$ at room temperature ${ }^{17}$ or on $\mathrm{MgO}$ at various temperatures. ${ }^{18}$ These works conclude that nucleation occurs preferentially on point defect sites rather than by random nucleation.

As opposed to these earlier works, PLD is a transient process. It has recently been reported that the average time of flight from target to substrate of neutral species ablated under similar conditions than in this work was close to $4 \mu$ s (Ref. 20) and that this average time varies little with fluence. ${ }^{21}$ Assuming $10 \mu \mathrm{s}$ as a conservative approximation for the deposition time in our conditions as was earlier done in Ref. 8 , the average flux calculated dividing the number of deposited atoms per pulse by this deposition time is in the range of $0.5-1.5 \times 10^{16}$ at. $\mathrm{cm}^{-2} \mathrm{~s}^{-1}$. Earlier works using nontransient techniques reported fluxes in the range of $10^{12}-10^{13}$ at. $\mathrm{cm}^{-2} \mathrm{~s}^{-1}, 5,17,18$ i.e., close to 3 orders of magnitude lower. Assuming that nucleation occurs at point defects (i.e., nucleation rate is linear in flux), ${ }^{5,22}$ using the scaling factor reported in Ref. 18, and provided that the higher the saturation value of the number density of NPs the higher the nucleation rate, we calculate a minimum nucleation rate in our case of $5 \times 10^{13} \mathrm{~cm}^{-2} \mathrm{~s}^{-1}$. This value is five times higher than the saturation number density extrapolated at room temperature from the Arrhenius dependence reported elsewhere for gold on $\mathrm{MgO}$ (100) produced by conventional evaporation techniques. ${ }^{5}$ This very high nucleation rate is most likely related to the very high number of defects created during the PLD growth process. It has recently been reported that the ionization fraction of the laser-produced gold plasma under similar fluence conditions than in this work is approximately $60 \%{ }^{21}$ It contains in addition a similar number of electrons since the plasma should be neutral. Therefore, more than half of the high flux refers to charged species bombarding the substrate with enough ions having energies well above 200 $\mathrm{eV} .{ }^{21} \mathrm{It}$ is interesting to point out here that the charge emitted during electron-beam deposition of gold generated surface defects that acted as preferred nucleation sites for gold, ${ }^{17}$ even when the flux was 3 orders of magnitude smaller than in the present work. Also, the use of ion beam deposition leads to intermediate values of the number density and thus an enhanced nucleation rate. ${ }^{19}$ It is thus concluded that the high nucleation rate achieved in PLD is controlled by the special features of the process, namely, by defects created during growth by high energetic species bombardment rather than by the nature, structure, or surface energy of the substrate.

A condition generally acknowledged for epitaxial growth to occur is that the lattice mismatch of film and substrate is small as it occurs for the case of $\mathrm{Au}$ on $\mathrm{MgO}$. In addition, epitaxial growth is considered to be characterized by $\boldsymbol{D} / \boldsymbol{F}$ $>10^{5}$ where $\boldsymbol{D}$ is the diffusion rate and $\boldsymbol{F}$ is the deposition flux. ${ }^{22,23}$ This relation technically means that it is achieved at high temperatures when $\boldsymbol{D}$ is high. This reasoning is however 
in contrast with very early observations of epitaxial growth of silver at low temperatures using sputtering and a deposition rate of $0.16 \mathrm{~nm} \mathrm{~s}^{-1}\left(F \approx 10^{15}\right.$ at $\left.\mathrm{cm}^{-2} \mathrm{~s}^{-1}\right) .{ }^{12}$ The high average kinetic energies of the species (10-50 times higher than in a thermal evaporation process) were considered responsible for the epitaxial growth on the bases of the species increased mobility. This reasoning has also been applied more recently to explain the oriented growth of Au NPs by PLD at room temperature. ${ }^{4,10}$ Furthermore, a comparison study of Fe produced by MBE and PLD on Mo(110) shows that the diffusion rate in the latter is 4 orders of magnitude higher leading to the same $\boldsymbol{D} / \boldsymbol{F}$ value at $300 \mathrm{~K}$ for both techniques. $^{24}$ The power law $\boldsymbol{D} / \boldsymbol{F} \approx \boldsymbol{l}^{6} / \boldsymbol{l n}\left(\boldsymbol{l}^{2}\right)$ relating the flux and diffusion rates with the mean island distance $\boldsymbol{l}$ (Refs. 23 and 24) allows us to estimate the diffusion rate in the case of $\mathrm{Au}$ on $\mathrm{MgO}$ produced by PLD in this work $\left[\boldsymbol{D}_{\mathrm{PLD}}(\mathrm{RT})\right.$, where RT stands for room temperature deposition] with respect to that reported earlier for the case of electron-beam evaporation at $381{ }^{\circ} \mathrm{C}\left(\boldsymbol{D}_{\mathrm{EB}}(T=381)\right) .{ }^{18}$ The mean separation in the latter case was $\boldsymbol{l}_{\mathrm{EB}}=9.5 \mathrm{~nm}$ whereas in our case, $\boldsymbol{l}_{\text {PLD }}$ can be determined as the center-to-center separation of NPs in Fig. 3(a) and is approximately $4.5 \mathrm{~nm}$, i.e., $\boldsymbol{l}_{\mathrm{EB}}(T=381) \approx 2 \boldsymbol{l}_{\mathrm{PLD}} \quad(\mathrm{RT})$. The comparison leads to $\boldsymbol{D}_{\mathrm{PLD}}(\mathrm{RT}) / \boldsymbol{D}_{\mathrm{EB}}(T=381)=2.5 \times 10^{2}$. If we assume that the activation energy for diffusion of $\mathrm{Au}$ on $\mathrm{MgO}$ is $0.2-0.3 \mathrm{eV},{ }^{25}$ $\boldsymbol{D}_{\mathrm{PLD}}(\mathrm{RT}) / \boldsymbol{D}_{\mathrm{EB}}(\mathrm{RT}) \approx 10^{4}-10^{5}$. This result agrees well with the earlier report for $\mathrm{Fe}$ on $\mathrm{Mo}(110)$ in spite of the very different systems studied, i.e., metal-metal in Ref. 24 and metal-oxide in this work. This allows the conclusion that the 4-5 orders of magnitude enhancement of diffusion rate is related to the special features of PLD rather than to the substrate used.

Generally, the equilibrium shape of large metal NPs on a support can be predicted from the Wulff-Kaichew theorem. For fcc structures, the equilibrium shape is a truncated octahedron in which the metal-oxide adhesion energy $\boldsymbol{E}_{\mathrm{ad}}$ to surface energy $\gamma_{\boldsymbol{I}}$ ratio determines the degree of truncation through the expression $\boldsymbol{E}_{\text {ad }} / \boldsymbol{\gamma}_{\boldsymbol{i}}=\boldsymbol{\Delta} \boldsymbol{h} / \boldsymbol{h}_{\boldsymbol{i}}$, where $\boldsymbol{h}_{\boldsymbol{i}}$ is the central distance to the facet parallel to the interface and $\boldsymbol{\Delta} \boldsymbol{h}$ is the degree of truncation. ${ }^{3}$ There are several reports providing similar values for the adhesion energy for $\mathrm{Au}$ on $\mathrm{MgO}$ (100), whereas the values reported for the surface energy of $\mathrm{Au}(100)$ have much higher dispersion. Using both data reported in Ref. 26, $\boldsymbol{E}_{\mathrm{ad}} / \boldsymbol{\gamma}_{I}=0.62$ and thus partial wetting is predicted by the Wulff-Kaichew theorem. This prediction is in agreement with our experimental results in Fig. 6 showing that the aspect ratio defined by the height to lateral dimension ratio is well below 1 . From the large crystal whose cross-section view is seen in Fig. 6(b), $\boldsymbol{\Delta} \boldsymbol{h} / \boldsymbol{h}_{\boldsymbol{i}} \approx 0.55$ within $20 \%$ that is in excellent agreement with the prediction of the Wulff-Kaichew theorem. When the NPs are smaller [see the one highlighted in Fig. 6(c)], they are typically octahedral with limited or not significant degree of truncation. This modification in shape is most likely due to the large fraction of edge sites for such small sizes as discussed elsewhere for the case of $\mathrm{Pd}$ on $\mathrm{MgO}^{3}$

\section{CONCLUSIONS}

Pulsed laser deposition of gold on amorphous (carbon/ glass) and single-crystalline $(\mathrm{MgO})$ substrates leads to similar deposition and nucleation rates irrespective of the nature of the substrate, supporting that nucleation is dominated by defects created by the large fraction of ions having energies higher than $200 \mathrm{eV}$ rather than by different surface energies of the substrates. A minimum nucleation rate of 5 $\times 10^{13} \mathrm{~cm}^{-2} \mathrm{~s}^{-1}$ has been estimated, which is much higher than that achieved with more standard evaporation techniques. Instead, the structure of the substrate plays an essential role on the oriented growth and shape of nanoparticles. The large flux of species promotes epitaxial growth on $\mathrm{MgO}$ at room temperature due to a diffusion rate that is $4-5$ orders of magnitude higher than in electron-beam evaporation. This leads to faceted nanoparticles with height to lateral dimension ratio $<0.7$ as opposed to those on amorphous substrates for which this ratio is $>0.7$. Furthermore, the shape of the large faceted NPs on $\mathrm{MgO}$ is well explained using WulffKaichew theorem. It is concluded that pulsed laser deposition in combination with single-crystalline substrates is a promising means to achieve control over the shape of metal NPs at room temperature.

\section{ACKNOWLEDGMENTS}

This work has partially been supported by EU (Grant No. HPRN-CT-2002-00328) and MEC (Spain) (Grant No. MAT2005-06508-C02-01). E. I. Kauppinen from Aerosol Technology Group in VTT Technical Research Centre (Espoo, Finland) and J. García from Centro Nacional de Aceleradores, Universidad de Sevilla and CSIC (Spain) are, respectively, acknowledged for providing the $\mathrm{MgO}$ nanocubes and the RBS measurements.

\footnotetext{
*Present address: ENEA, Department of Advanced Physical Technologies and New Materials, Brindisi Research Center, S.S. 7 Appia km 706, I-72100 Brindisi, Italy.

${ }^{1}$ J. R. Lakowicz, Plasmonics 1, 5 (2006).

${ }^{2}$ G. Glaspell, H. M. A. Hassan, A. Elzatahry, L. Fuoco, N. R. E. Radwan, and M. S. El-Shall, J. Phys. Chem. B 110, 21387 (2006).

${ }^{3}$ C. R. Henry, Prog. Surf. Sci. 80, 92 (2005), and references therein.
}

${ }^{4}$ A. Zenkevitch, J. Chevallier, and I. Khabelashvili, Thin Solid Films 311, 119 (1997).

${ }^{5}$ K. Hojrup-Hansen, S. Ferrero, and C. R. Henry, Appl. Surf. Sci. 226, 167 (2004).

${ }^{6}$ C. Revenant, G. Renaud, R. Lazzari, and J. Jupille, Nucl. Instrum. Methods Phys. Res. B 246, 112 (2006).

${ }^{7}$ Pulsed Laser Deposition of Thin Films, edited by R. Eason (Wiley Interscience, New York, 2007).

${ }^{8}$ J. Gonzalo, A. Perea, D. Babonneau, C. N. Afonso, N. Beer, J.-P. 
Barnes, A. K. Petford-Long, D. E. Hole, and P. D. Townsend, Phys. Rev. B 71, 125420 (2005).

${ }^{9}$ R. Serna, J. Gonzalo, A. Suarez-Garcia, C. N. Afonso, J. P. Barnes, A. K. Petford-Long, R. C. Doole, and D. Holes, J. Microsc. 201, 250 (2001).

${ }^{10}$ E. Irissou, B. Le Drogoff, M. Chaker, and D. Guay, J. Appl. Phys. 94, 4796 (2003).

${ }^{11}$ R. Dolbec, E. Irissou, M. Chaker, D. Guay, F. Rosei, and M. A. El Khakani, Phys. Rev. B 70, 201406 (2004).

${ }^{12}$ K. L. Chopra and R. Randlett, Appl. Phys. Lett. 8, 241 (1966).

${ }^{13}$ R. Grisel, K. J. Weststrate, A. Gluhoi, and B. E. Nieuwenhuys, Gold Bull. 35, 39 (2002).

${ }^{14}$ B. Pauwels, G. Van Tendeloo, W. Bouwen, L. T. Kuhn, P. Lievens, H. Lei, and M. Hou, Phys. Rev. B 62, 10383 (2000).

${ }^{15}$ I. S. Altman, P. V. Pikhitsa, M. Choi, H.-J. Song, A. G. Nasibulin, and E. I. Kauppinen, Phys. Rev. B 68, 125324 (2003).

${ }^{16}$ H. Hofmeister, J. Optoelectron. Adv. M. 9, 99 (2007).
${ }^{17}$ J. M. Mativetsky, S. Fostner, S. A. Burke, and P. Grutter, Surf. Sci. 602, L21 (2008).

${ }^{18}$ J. L. Robins and T. N. Rhodin, Surf. Sci. 2, 346 (1964).

${ }^{19}$ S. Muzard, C. Templier, J. Delafond, J. C. Girard, D. Thiaudiere, L. Pranevicius, and A. Galdikas, Surf. Coat. Tech. 100-101, 98 (1998).

${ }^{20}$ J. Gonzalo, J. Siegel, A. Perea, D. Puerto, V. Resta, M. GalvanSosa, and C. N. Afonso, Phys. Rev. B 76, 035435 (2007).

${ }^{21}$ A. Perea, J. Gonzalo, C. Budtz-Jorgensen, G. Epurescu, J. Siegel, C. N. Afonso, and J. Garcia-Lopez, J. Appl. Phys. 104, 084912 (2008).

${ }^{22}$ J. A. Venables, Surf. Sci. 299/300, 798 (1994).

${ }^{23}$ H. Brune, Surf. Sci. Rep. 31, 121 (1998).

${ }^{24}$ P. O. Jubert, O. Fruchart, and C. Meyer, Surf. Sci. 522, 8 (2003).

${ }^{25}$ G. Barcaro and A. Fortunelli, New J. Phys. 9, 22 (2007).

${ }^{26}$ L. M. Molina and B. Hammer, Phys. Rev. Lett. 90, 206102 (2003). 